\title{
Rediseño del sistema de transporte de racimos de banano para la recolección en el campo
} Caso de estudio: Studer Innotec Redesign of the transport system of banana bunches for field harvesting

\section{Case study: Studer Innotec}

Jose Luis Ordoñez-Avila ${ }^{1}$, Maria Elena Perdomo-Perdomo²

Ordoñez-Avila. J; Perdomo-Perdomo, M. Rediseño del sistema de transporte de racimos de banano para la recolección en el campo. Caso de estudio: Studer Innotec. Tecnología en Marcha. Vol. 32-4. Octubre-Diciembre 2019. Pág 171-178.

doi) https://doi.org/10.18845/tm.v32i4.4801

1 Máster en Administración de Proyectos profesor investigador, Facultad de Ingeniería y Arquitectura de la Universidad Tecnológica Centroamericana. Honduras. Correos electrónicos:c, jlord_1987@hotmail.com. 


\section{Palabras clave}

Agroindustrial; Batería de litio; Inversores; Logística; Postcosecha.

\section{Resumen}

El presente trabajo describe la experiencia de integración tecnológica en el caso de estudio de Studer Innotec, compañía que fabrica inversores/cargadores y reguladores en Suiza, utilizando un inversor/cargador se rediseño el sistema de transporte mejorando la eficiencia de la cadena agro-productiva de la post cosecha del banano, desarrollado en el sector atlántico de Honduras. Actualmente el sistema de transporte de banano se realiza en cable vía utilizando animales de carga (mulas) haciendo un proceso lento, además de generar costos por el cuido de estos animales o por máquinas subidas al cable vía que utilizan un motor de combustión diésel acoplado a una bomba de aceite, los cuales generan emisiones de gases que contaminan el ambiente e incluso el mismo banano.

Con el objetivo de integrar equipos industriales que permitan recolectar una mayor cantidad de racimos que en el sistema actual, asegurando las propiedades de calidad del banano se presenta el rediseño de una maquina utilizadas para el transporte del banano, cambiando su motor diésel de $9 \mathrm{hp}$, por un motor trifásico de $5 \mathrm{hp}$, donde el mayor reto ha sido pasar la energía eléctrica almacenada en baterías por un inversor, hasta llegar a un variador de frecuencia, con el que se controló el torque y velocidad del motor, permitiendo un mejor uso del equipo con lo que se ha logrado transportar más de 70 racimos de banano en un recorrido mayor a 800 metros dentro de la finca.

\section{Keyworks}

Agroindustry; Lithium battery; Investors; Logistics; Post-harvest.

\section{Abstract}

This paper describes the experience of technological integration in the case study of Studer Innotec, company that manufactures inverters / chargers and regulators in Switzerland, using an inverter / charger to redesign the transport system improving the efficiency of the agro-productive chain of the post-harvest of banana, developed in the Atlantic sector of Honduras. Currently, the banana transport system is carried out via cable using load animals (mules) making a slow process, in addition to generating costs for the care of these animals or by machines uploaded to the cable via a diesel combustion engine coupled to an oil pump, which generate emissions of gases that pollute the environment and even the banana itself.

With the aim of integrating industrial equipment that allows to collect a larger number of bunches than in the current system, ensuring the quality properties of the banana is presented the redesign of a machine used to transport the banana, changing its diesel engine of $9 \mathrm{hp}$, by a 5-hp three-phase motor, where the greatest challenge has been to pass the electric energy stored in batteries by an inverter, until reaching a frequency inverter, which controlled the torque and speed of the motor, allowing a better use of the motor, with which it has been possible to transport more than 70 bunches of banana in a route greater than 800 meters inside the farm. 


\section{Introducción}

Por años Honduras ha sido líder en la producción y exportación de banano, siendo el tercer producto que más se exporta en la actualidad, para el 2017 las exportaciones de banano fueron de 182.2 (millones de dólares), ubicándose en el tercer lugar, después del camarón y el café [1].

Las plantas de procesamiento de banano llevan a cabo su proceso desde el cultivo, recolección y empaque de este, los cuales son exportados a los mercados internacionales como Estados Unidos entre otros; estas plantas procesadoras representan una importante fuente de empleo para nuestro país. Estos procesos cada día sufren cambios, obligando a las empresas a estar en la constante búsqueda de mejora para sus procesos, que tengan como resultado una respuesta rápida a esas necesidades cambiantes del mercado. Como lo menciona Deming [2] "Ios clientes siempre buscan excelencia en la calidad, precios accesibles y velocidad de respuesta en sus necesidades entre todo".

Actualmente en la mayoría de las empresas cosechadoras de banano en Honduras se utiliza un sistema de empuje empírico de los racimos de banano, a través de un animal de carga. Esto conlleva a la dependencia del animal y la falta de mejora en la eficiencia del sistema. Además, el conjunto de tecnología que acompaña el sistema de empuje actual requiere de un estudio de verificación de calidad técnica. Justificándose la necesidad de aprovechar una innovación más tecnológica que requiera modificar algunas estructuras parciales o equipos que componen actualmente el sistema de carga.

Las actividades de transporte generalmente representan un elemento individual importante que tiene un gran impacto en los costos de logística para la mayoría de las empresas. Se ha observado que el movimiento de carga en todas las etapas del proceso absorbe entre uno y dos tercios de los costos totales de logística. Por ello los responsables de logística necesitan comprender y buscar alternativas que mejoren la eficiencia en las actividades de transportación [3].

Este caso de estudio se basó en el análisis y la mejora del sistema de transporte del banano desde las fincas hasta las plantas de empaque de este. Actualmente el sistema de transporte del banano se realiza en un cable vía que está distribuido en toda la finca que se conecta hasta la planta empacadora y es acarreado por un animal de carga (mula). Mostrando que los sistemas de transporte de banano carecen de automatización, por lo que el objetivo de esta investigación fue rediseñar una máquina para transportar el banano, utilizando equipos europeos de alto rendimiento, los cuales vuelven más eficiente el proceso de recolección de banano, teniendo en cuenta los estándares de seguridad industrial y calidad entre otros.

\section{Materiales y métodos}

Los programas de vinculación e investigación de la Universidad Tecnológica Centroamericana (UNITEC) permiten a los alumnos ser parte de proyectos donde se diseñan maquinas e integran tecnología para poder apoyar al desarrollo agroindustrial del país, respondiendo a diferentes necesidades como ser la recolección del banano.

La metodología que se utilizó se basó en que los diseñadores industriales reutilizan la información, no se comienza un diseño desde cero, sino que se inicia mejorando las características de un modelo físico ya existente, mejorando el prototipo [4].

Para tal efecto, se rediseño e integro una maquina basándose en las dimensiones de la máquina en las plantas cosechadoras y poder realizar cambios en busca del diseño que se ajustó a las necesidades actuales de la finca. También se realizó un análisis de la operación, en el cual se tomaron en cuenta una descripción del proceso de transporte, donde sobresalen la cantidad de 
60 racimos que puede traer con la mula y la existencia de una maquina obsoleta con un motor diésel de $9 \mathrm{hp}$ con 3500 revoluciones por minuto.

Los equipos instalados con los cuales se sustituyó un motor de combustión fueron: baterías de litio con estructura química LiFePO4, estas se han constituido en el producto de mayor interés comercial como material catódico de baterías de ion litio, fundamentalmente por sus características amigables al medio ambiente, bajo costo de sus precursores y larga vida en el proceso de ciclización [5], un inversor que podemos definir como un sistema que sin necesidad de estar conectados a ninguna red alterna, permite transformar corriente continua a alterna [6], un variador de frecuencia para controlar un motor trifásico de $5 \mathrm{hp}$ el cual se conectó a la bomba hidráulica de la maquina actual.
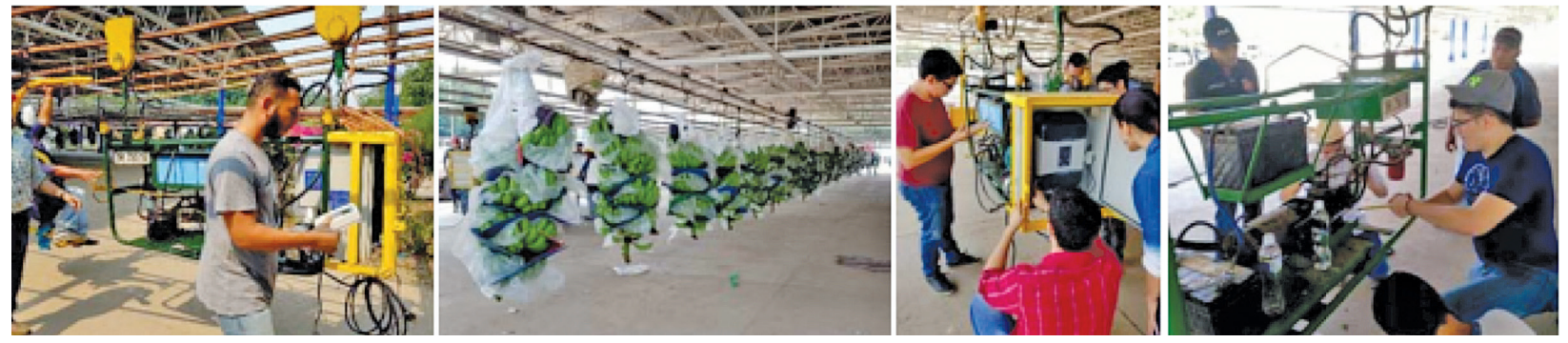

Figura 1. Instalación en planta cosechadora. [7]

Fue necesario la compra de equipos europeos ya que el mercado nacional no tenía acceso a equipos con las características necesarias para el desarrollo del proyecto. Cuando se obtuvieron los equipos se procedió de la siguiente manera: instalación de los equipos en el laboratorio de mecatrónica (UNITEC), pruebas de laboratorio a para determinar la autonomía de las baterías, rediseño y mecanización de estructura y finalmente pruebas de campo.

Una de las ventajas del inversor y variador fue la posibilidad de monitorear las baterías y el motor por lo que es posible determinar un plan de mantenimiento preventivo basado en predicciones, monitoreando las condiciones en las que se encuentra la maquinaria para buscar predecir cuándo será necesario parar con el propósito de realizarle mantenimiento [8], evitando daños o mal funcionamiento que pongan en peligro al personal, al banano o las instalaciones.

\section{Resultados y discusión}

Inicialmente se instalaron los equipos en el laboratorio basado en el diagrama de conexión (figura 2) con el cual se colocan 48 V DC hasta controlar un motor trifásico AC 220 V. Gracias a que el equipo cuenta con un dispositivo que almacena información en una SD permitió la realización de pruebas de laboratorio para comprender el comportamiento y autonomía de las baterías y a su respectiva documentación.

Se comenzó las pruebas de laboratorio con la finalidad de revisar el funcionamiento de los equipos haciendo girar el motor son carga, el tiempo de respuesta de las baterías es mayor a 11 horas el voltaje de las baterías en su punto más bajo (52.16V) está lejos del voltaje de descarga (48V). Al no tener una carga en el motor y no existir perturbaciones en el sistema el consumo energético es estable teniendo como promedio en la prueba de 11 horas, un consumo de 0.325kVA y un máximo de 0.37kVA. 


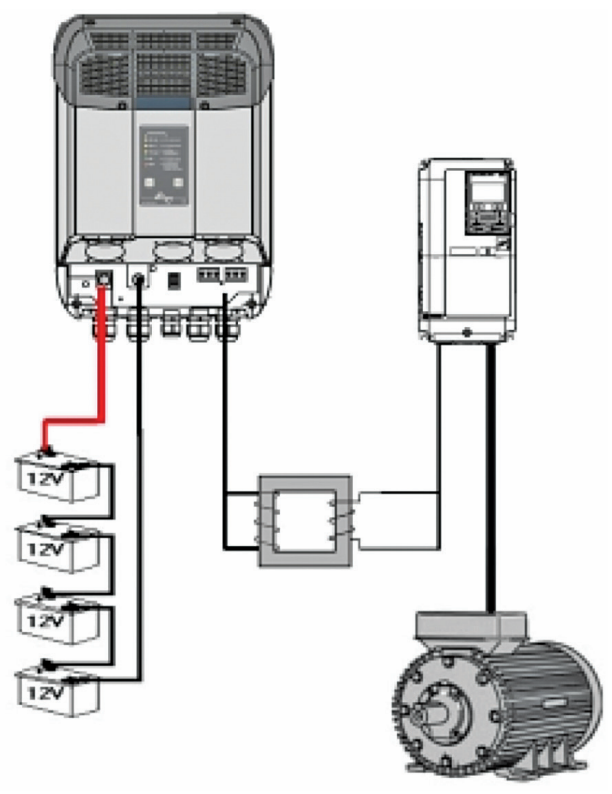

Figura 2. Diagrama de conexión de los dispositivos.

Posteriormente se giró el motor con el equipo hidráulico a una frecuencia de 35 Hertz y se generan perturbaciones encendiendo y apagando el motor donde se verifico las rampas de aceleración y desaceleración configuradas en el variador, el tiempo de respuesta de las baterías se disminuye a 4 horas, la gráfica (figura 3) muestra que el voltaje de las baterías en su punto más bajo (47.8V) después de 4 horas con 15 minutos de trabajo está por debajo del voltaje de descarga (48V). Disminución de aproximadamente $1.125 \mathrm{~V}$ por hora.

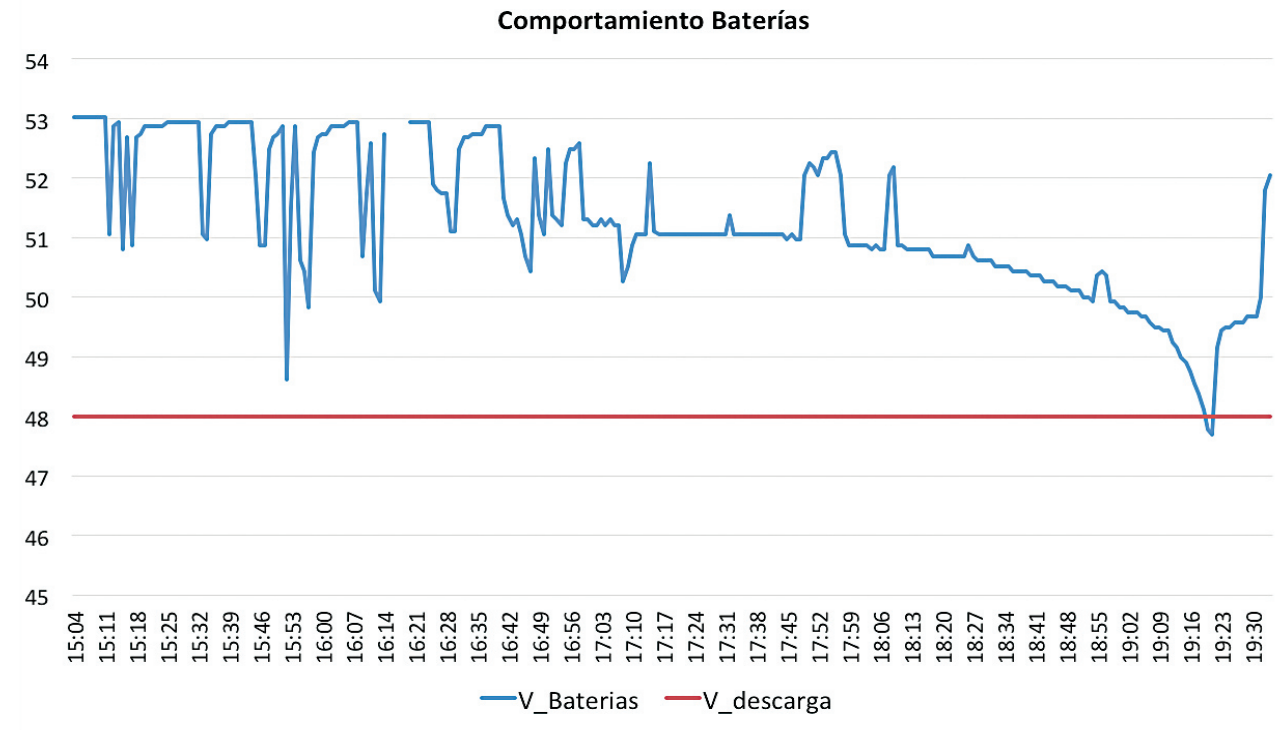

Figura 3. Voltaje de baterías en relación con el tiempo de descarga.

Pese a que el promedio de consumo se mantuvo a lo largo de la prueba 0.332kVA, los picos llegaron a un máximo de 2.03kVA, provocando la descarga de las baterías en 4 horas 15 (figura. 4). 


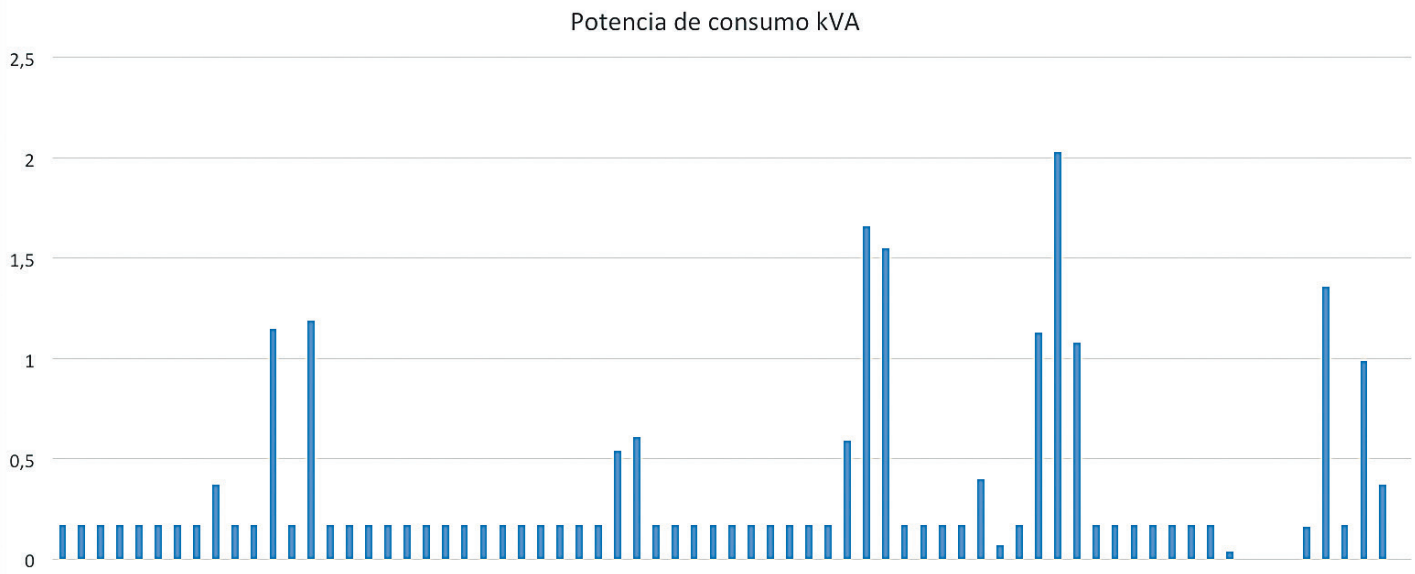

Figura 4. Consumo de potencia durante el tiempo de descarga.

Finalmente cargamos las baterías de 7:30 pm a 10:00 pm. La carga se realizó a 25 A. El equipo tiene capacidad para ser cargado a $50 \mathrm{~A}$, por lo que se estimó que las baterías se cargaran en 1 hora con 15 minutos. Esta configuración se realizó para cargar las baterías en la planta cosechadora. Pese a que la prueba se realizó cargando las baterías a la red eléctrica, es posible por la naturaleza del proyecto utilizar energía solar, al igual como lo menciona Guzmán [9] "el porcentaje de la energía del sol que llega directamente a la Tierra se aprovecha en alguna medida para producir corriente eléctrica mediante celdas fotovoltaicas" y de esta forma cargar las baterías.

Mientras se realizaban las pruebas en el laboratorio se procedió con el diseño industrial basándonos en el grado de funcionalidad que es el factor que marca la mayor parte de los objetivos que se pretenden cubrir, es decir, es el punto estratégico del diseño [4], lo que nos conlleva a tener en cuenta que el diseño que se realizo fue el más funcional posible entendiéndose como funcional protección al equipo, usabilidad y ergonomía del mismo, dejando atrás aspectos estéticos y costo. Al terminar las pruebas de laboratorio de forma congruente se procedió a rediseñar en software CAD el equipo y montar los dispositivos en la estructura.

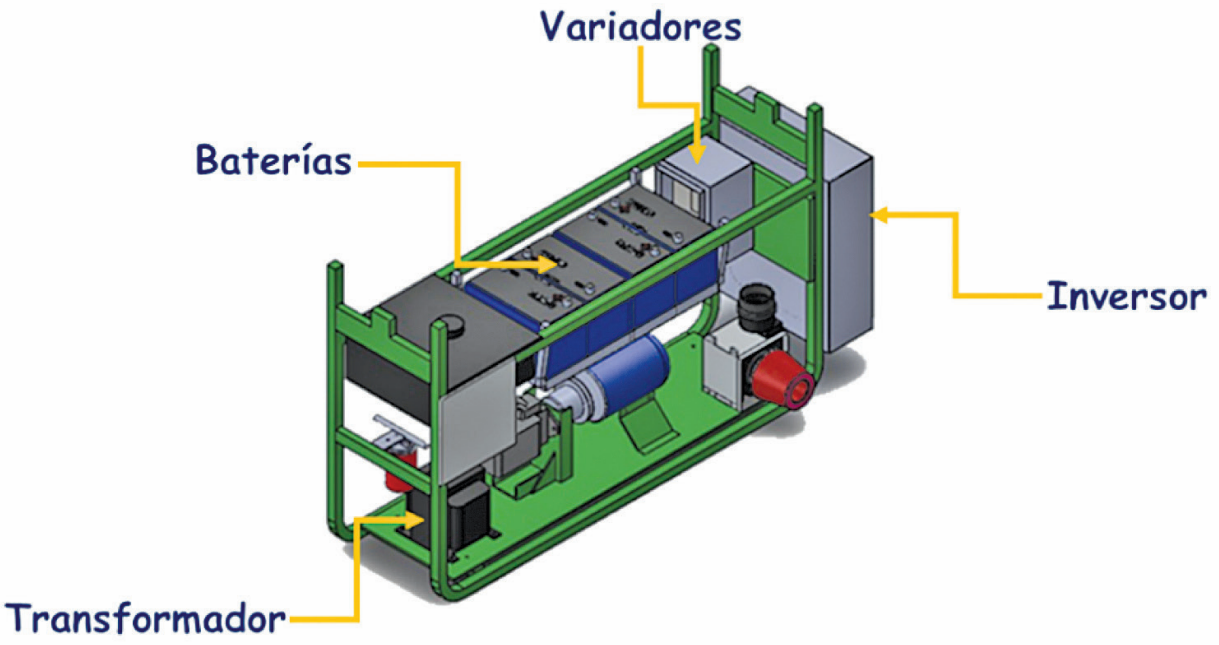

Figura 5. Diseño final en software CAD. 
La prueba de campo se realizó inicialmente con 70 racimos de banano, los cuales fueron transportados 400 metros por el cable vía. El voltaje de las baterías disminuyo en $0.5 \mathrm{~V}$, el recorrido duro aproximadamente 40 minutos. EL consumo máximo fue de $2.88 \mathrm{kVA}$ y la frecuencia de trabajo fue de 25 Hertz. Posteriormente cuando se realizaron pruebas con 100 racimos se notó que era necesario aumentar el torque en el arranque hasta que este fuera capaz de mover los racimos y posteriormente aumentar la velocidad. Esto se realizó configurando el uso de multi velocidades en el variador lo cual permitió que el arranque de la maquina se realice a 20 Hertz y posteriormente se aumente mediante los interruptores adicionales para 30 y 35 Hertz.

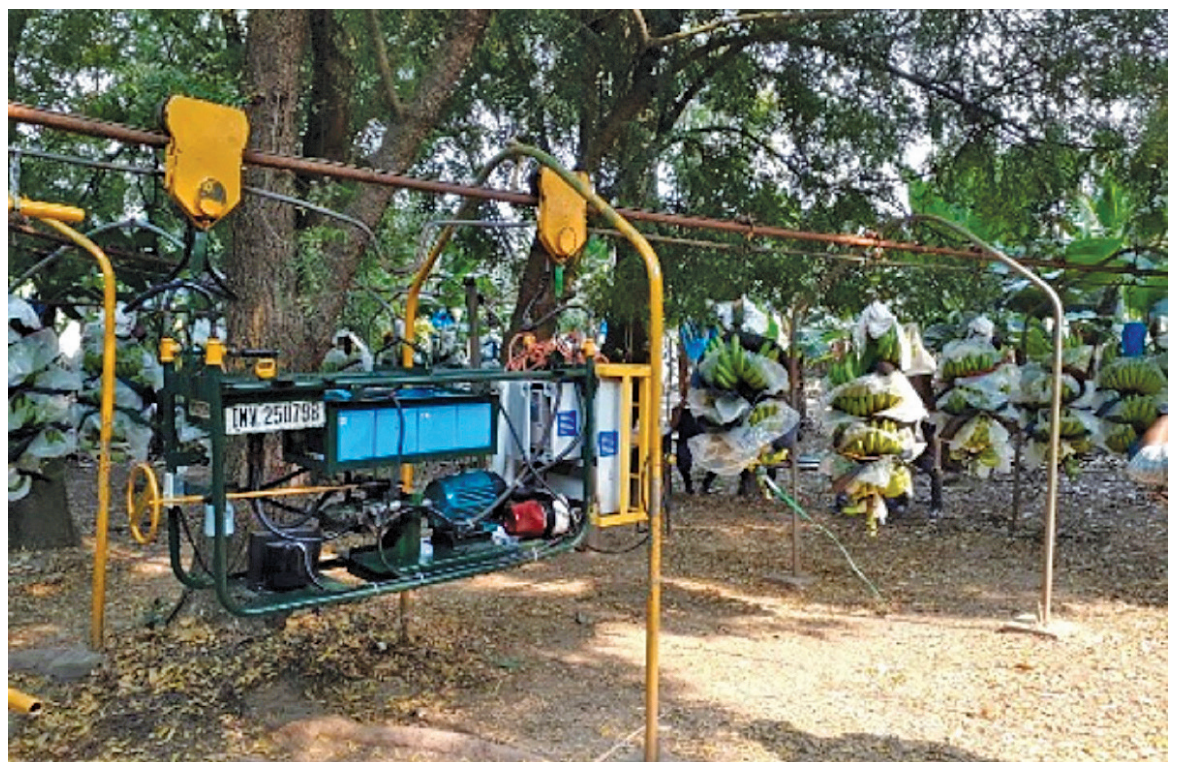

Figura 6. Prototipo terminado y prueba en campo.

Los equipos europeos que se utilizaron para rediseñar esta máquina son robustos, capaces de trabajar en distintas aplicaciones. Como lo menciona Studer Innotec en su caso de estudio [7], "el integrar el inversor en este proyecto, permitió que el sistema tenga una alimentación de energía estable. Además, que dio flexibilidad en su programación permitiendo optimizar el uso de la carga y descarga de las baterías".

\section{Conclusión}

De acuerdo con los resultados obtenidos en la planta cosechadora de banano en el sector atlántico de Honduras, y basados en que el análisis por medio de la evaluación física es vital para verificar el correcto diseño de un producto o prototipo [4], podemos afirmar que el sistema diésel y los animales de arrastre, se podrán sustituir por 4 baterías de litio, un inversor, un variador de frecuencia y un motor trifásico, logrando la movilización de racimos de banano en mayor cantidad que con el sistema tradicional. Aunado a ello, el uso de esta tecnología disminuye la generación de gases de efecto invernadero que afectan el cambio climático.

Por otro lado, queda abierta la investigación para el desarrollo de una maquina más inteligente la cual tenga capacidades de ser utilizada sin operador y esta pueda recorrer la finca de forma autónoma, buscando tener un mayor grado de automatización, precisión e incluso inteligencia para mejorar el consumo de energía y aumentar la autonomía del dispositivo. 


\section{Referencias}

[1] I. N. d. Estadisticas, «http://www.ine.gob.hn/,» 8 Mayo 2018. [En línea]. Available: http://www.ine.gob.hn/index. php?option=com_content\&view=article\&id=168.

[2] C. C. Sornoza, Gestion de la calidad, Mexico: Pearson, 2011.

[3] R. Ballou, Logistica, Administración de la Cadena de Suministros, Mexico: Pearson, 2004.

[4] L. R. y. M. D. Lucía Rodríguez Parada, «Modelo de análisis y validación de propuestas de diseño mediante prototipos flexibles,» DYNA - Ingeniería e Industria , vol. 91, n 5, p. 502, 2017.

[5] F. B. M. V. J. L. F. M. O. J. V. R. M. N. L. M. L. W. Y. M. B. E. R. P. R. B. Saúl Cabrera, «PERSPECTIVAS EN EL PROCESAMIENTO DE MATERIALES - ELECTRODOS PARA BATERÍAS DE ION LITIO EN BOLIVIA,» REVISTA BOLIVIANA DE QUÍMICA, vol. 29, n 1, p. 22, 2012.

[6] J. A. Castillo, «Diseño de un inversor trifásico de siete niveles controlado con máquina de estados,» Tecnología en Marcha, vol. 30, n 2, p. 89, 2017.

[7] S. Innotec, «http://www.studer-innotec.com,» 2305 2018. [En línea]. Available: http://www.studer-innotec.com/ media/document/0/honduras-movimiento-de-bananos-dole.pdf.

[8] J. R. Ocampo, "SISTEMA DE BAJO COSTO PARA MONITOREO DE DISTURBIOS DE VIBRACIÓN EN MÁQUINAS ROTATORIAS,»Innovare, vol. 2, n² 2, p. 15, 2013.

[9] T. Guzmán-Hernández, F. Araya-Rodríguez, G. Castro-Badilla, y J. Obando-Ulloa, «Uso de la energía solar en sistemas de producción agropecuaria: producción más limpia y eficiencia energética,» Tecnologia en Marcha, vol. 29, n 8, p. 48, 2016. 